\title{
A BOUND FOR THE NUMBER OF AUTOMORPHISMS OF A COMPACT KLEIN SURFACE WITH BOUNDARY
}

\author{
COY L. MAY
}

\begin{abstract}
For an integer $g \geqslant 2$, let $\nu(g)$ be the order of the largest group of automorphisms of a compact Klein surface with nonempty boundary of genus $g$. We show that $\nu(g) \geqslant 4(g+1)$ for all $g$ and that for an infinite number of values of $g, \nu(g)=4(g+1)$.
\end{abstract}

0. Introduction. Let $X$ be a Klein surface [2], that is, $X$ is a surface with boundary $\partial X$ together with a dianalytic structure on $X$. The surface $X$ need not be orientable. A homeomorphism $f: X \rightarrow X$ of $X$ onto itself that is dianalytic will be called an automorphism of $X$.

In a recent paper [7], we showed that a compact Klein surface with nonempty boundary of algebraic genus $g \geqslant 2$ cannot have more than $12(g-1)$ automorphisms. After [7] appeared we learned that the upper bound for orientable surfaces with boundary had been obtained earlier by Oikawa [10]. Both [7] and [10] use techniques which go back to the fundamental paper of Hurwitz [4]. The bound $12(g-1)$ is attained for both orientable and nonorientable surfaces.

In special cases it is possible to improve the upper bound. In fact, for certain topological types of orientable surfaces with boundary, the order of the largest possible group of orientation-preserving automorphisms has been determined. This has been done recently for orientable surfaces with three or less boundary components by Kato [5] and much earlier for surfaces of low topological genus. The cases of topological genus 0,1 , and 2 (orientable with boundary) were treated by Heins [3], Oikawa [10], and Tsuji [12] respectively.

Here we shall be interested in the size of the full automorphism group. For an integer $g \geqslant 2$, let $\nu(g)$ be the order of the largest group of automorphisms of a compact Klein surface with nonempty boundary of algebraic genus $g$. Then $\nu(g) \leqslant 12(g-1)$ for all $g$. We have shown that $\nu(g)=12(g-1)$ for an infinite number of values of the genus $g$ [8]. However, we also proved that $\nu(g)<12(g-1)$ for infinitely many values of $g[9]$.

Received by the editors January 19, 1976.

AMS (MOS) subject classifications (1970). Primary 30A46, 14H30; Secondary 57A05.

Key words and phrases. Klein surface, automorphism group, algebraic genus, complex double, Riemann surface, ramified covering, Hurwitz ramification formula.

(1) American Mathematical Society 1977 
In this paper we establish a lower bound for $\nu(g)$. The corresponding result for Riemann surfaces was obtained independently by Accola [1] and Maclachlan [6].They showed that the best possible lower bound for the order of the largest group of automorphisms of a Riemann surface of genus $g \geqslant 2$ is $8(g+1)$.

We show that $\nu(g) \geqslant 4(g+1)$ for all $g$, and in fact $\nu(g)=4(g+1)$ for an infinite number of values of $g$. First for each $g$ we construct a surface with boundary that has an automorphism group of order $4(g+1)$. Then we show that for certain values of $g$ no larger group is possible. We use the methods of [1], [7], and [9].

Finally we obtain some further information about nonorientable surfaces. Our work shows that for certain values of $g$, a nonorientable surface with boundary of genus $g$ has at most $4 g$ automorphisms. We also construct for each $g$ a nonorientable surface with boundary that has an automorphism group of order $4 g$.

1. Fundamental definitions. If $X$ is a Klein surface, let $X^{\circ}=X-\partial X$. $X^{\circ}$ will be called the interior of $X$.

Let $X$ and $Y$ be Klein surfaces. A morphism [2, p. 17] is a continuous map $f: X \rightarrow Y$, with $f(\partial X) \subset \partial Y$ and the following local behavior. For every point $p \in X$ there exist dianalytic charts $(U, z)$ and $(V, w)$ at $p$ and $f(p)$ respectively such that $f(U) \subset V$ and $\left.f\right|_{U}=w^{-1} \circ \varphi \circ F \circ z$, where $F$ is an analytic function on $z(U)$ and $\varphi$ is the folding map, $\varphi(x+y i)=x+|y| i$.

Let $f: X \rightarrow Y$ be a nonconstant morphism of Klein surfaces. Let $x \in X$, and let $e_{f}(x)$ denote the ramification index of $f$ at $x$ [2, pp. 27-30]. $f$ is ramified at $x$ if $e_{f}(x)>1$; otherwise $f$ is unramified at $x$. Let $d_{f}(x)$ be the relative degree of $x$ over $f(x) \cdot d_{f}(x)=2$ if $x \in X^{\circ}$ and $f(x) \in \partial Y$; otherwise $d_{f}(x)=1$. The morphism $f$ will be called an $r$-sheeted covering of $Y$ if for every point $y \in Y$,

$$
\sum_{x \in f^{-1}(y)} e_{f}(x) \cdot d_{f}(x)=r
$$

Now let $X$ be a compact Klein surface. Let $\left(X_{c}, \pi, \sigma\right)$ be the complex double of $X$, that is, $X_{c}$ is a compact Riemann surface, $\pi: X_{c} \rightarrow X$ is an unramified 2sheeted covering of $X$, and $\sigma$ is the unique antianalytic involution of $X_{c}$ such that $\pi=\pi \circ \sigma$. The complex double is unique up to analytic isomorphism. For more details, see [2, pp. 37-40]. The complex double is particularly useful because it allows us to pull down results about Riemann surfaces to Klein surfaces.

Let $E$ be the field of all meromorphic functions on the compact Klein surface $X . E$ is an algebraic function field in one variable over $\mathbf{R}$ and as such has an algebraic genus $g$, i.e., the nonnegative integer that makes the algebraic version of the Riemann-Roch Theorem work. We will refer to $g$ simply as the genus of the compact Klein surface $X$. In case $X$ is a Riemann surface, $。$ equal to the topological genus of $X$. 
Henceforth by a surface we shall mean a compact surface.

2. An example. For any integer $g \geqslant 2$, let $X$ be a sphere with $g+1$ holes, with the holes placed around the equator, centered around the vertices of an inscribed regular polygon with $g+1$ sides. $X$ is an orientable Klein surface of genus $g$. $X$ has a group of orientation-preserving automorphisms isomorphic to the dihedral group $D_{g+1}$. Reflection in the plane of the equator is an orientation-reversing automorphism. Thus $X$ has $4(g+1)$ automorphisms. The automorphism group of $X$ is $C_{2} \times D_{g+1}$, where $C_{2}$ denotes the group of order two.

Consequently $\nu(g) \geqslant 4(g+1)$. For $g=2$ this example gives a group of maximum possible order.

3. Sharpness of the bound $4(g+1)$. Now we show that the bound $4(g+1)$ is the best possible. Our approach was inspired by Accola's treatment of the Riemann surface case [1].

If $G$ is a finite group, then denote the order of $G$ by $o(G)$.

Let $X$ be a Klein surface with nonempty boundary of genus $g \geqslant 2$. Let $G$ be a group of automorphisms of $X$. Then the quotient space $\Phi=X / G$ is a Klein surface with boundary, and the quotient map $\pi: X \rightarrow \Phi$ is a ramified $r$ sheeted covering of $\Phi$, where $r=o(G)$. For more details, see [2, pp. 52-56] and [7, pp. 200-203].

Let $p \in \Phi$. We will refer to the set $\pi^{-1}(p)$ as the fiber above $p$. Note that either $\pi^{-1}(p) \subset X^{\circ}$ or $\pi^{-1}(p) \subset \partial X$.

Now the quotient map $\pi: X \rightarrow \Phi$ is ramified above a finite number of points of $\Phi$, say $a_{1}, \ldots, a_{\omega}$. Let $k_{i}$ denote the ramification index of any point in the fiber above $a_{i}$. Let $n_{i}=1$ if $a_{i} \in \partial \Phi$, and let $n_{i}=2$ it $a_{l}-\Phi^{\circ}$. Let $\gamma$ denote the genus of the quotient space $\Phi$. Then the Hurwitz ramification formula for morphisms of Klein surfaces with boundary [7, p. 203] can be written

$$
\frac{2 g-2}{o(G)}=2 \gamma-2+\sum_{i=1}^{\omega} n_{i}\left(1-\frac{1}{k_{i}}\right) \text {. }
$$

We shall use this notation to state Lemmas 1-5.

Let $\left(X_{c}, f, \sigma\right)$ and $\left(\Phi_{c}, h, \tau\right)$ denote the complex doubles of $X$ and $\Phi$ respectively. Then $G$ is isomorphic to a group of orientation-preserving automorphisms of $X_{c}$ [2, p. 79]. Let $G$ act on $X_{c}$. Then we have the following result [9].

Lemma 1. The quotient space $X_{c} / G=\Phi_{c}$, and if $\tilde{\pi}: X_{c} \rightarrow \Phi_{c}$ denotes the quotient map, the following diagram commutes:

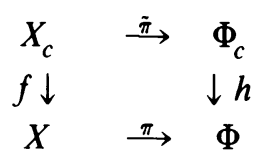

Next we give two simple applications of the Hurwitz ramification formula. 
LEMMA 2. If $\gamma \geqslant 1$, then $o(G) \leqslant 4(g-1)$.

For a proof, see [7].

LEMMA 3. If $G$ is abelian and $\gamma \geqslant 1$, then $o(G) \leqslant 2(g-1)$.

Proof. If $\gamma \geqslant 2$, then $(2 g-2) / o(G) \geqslant 2$ and $o(G) \leqslant g-1$. Suppose $\gamma=1$. Then $\omega \neq 0$ since $g \geqslant 2$. Let $G$ act on the complex double $X_{c}$ as in Lemma 1 . The covering maps $f$ and $h$ are unramified. If $\pi$ is ramified above a point $p \in \Phi$, then $\tilde{\pi}$ is ramified above each point in the fiber $h^{-1}(p)$. There is one point in the fiber $h^{-1}(p)$ if $p \in \partial \Phi$; otherwise there are two. Since $G$ is abelian, the quotient map $\tilde{\pi}$ is not ramified above precisely one point $[1, \mathrm{p}$. 403]. Hence if $\omega=1, \pi$ is ramified above an interior point $a_{1} \in \Phi^{\circ}$ and $n_{1}=2$; otherwise $\omega \geqslant 2$. In any case $(2 g-2) / o(G) \geqslant 0+2\left(1-\frac{1}{2}\right)$. Thus $o(G) \leqslant 2(g-1)$.

LEMma 4. Suppose $g-1=p$ where $p$ is a prime larger than 5 such that $3 \nmid(p-1)$. Then $o(G) \leqslant 8(g-1)$.

Proof. One of the results of [9] is that for such a value of $g, o(G)$ $<12(g-1)$. It can be shown by a calculation from [7, p. 207] that if $o(G)<12(g-1)$, then $o(G) \leqslant 8(g-1)$.

LEMMA 5. Suppose $g-1=p$ where $p$ is a prime larger than 5. Suppcse $G$ is cyclic of order $p$. Then the quotient map $\pi: X \rightarrow \Phi$ is unramified and $\gamma=2$.

Proof. Let $G$ act on the complex double $X_{c}$. Then by a result of Accola [1, p. 404], the quotient map $\tilde{\pi}$ is unramified. Therefore $\pi$ is unramified. Now $2(g-1) / p=2=2 \gamma-2$ and $\gamma=2$.

Actually the proof in [1, p. 404] of the corresponding result for Riemann surfaces carries over to the Klein surface case.

The next lemma is a special case of the main result of [9].

LEMMA 6. Let $Y$ be a Klein surface with boundary of genus 2 with a group of automorphisms $H$. If $q$ is a prime factor of $o(H)$, then either $q=2$ or $q=3$.

Proof. The only cyclic groups that can act on $Y$ are of order 6 or of order less than 5 [9]. Therefore the only possibilities for $q$ are 2 and 3.

LEMMA 7. Let $X$ be a Klein surface with boundary with a group of automorphisms $G$. Suppose $N$ is a normal subgroup of $G$. Then the factor group $H=G / N$ acts as a group of automorphisms of $X / N$ and $(X / N) / H=X / G$.

The corresponding result for Riemann surfaces is well known. The proof for Klein surfaces is similar and is omitted.

THEOREM 1. Let $p$ be a prime such that $p \equiv 11(\bmod 12)$. Let $g-1=p$. Let $X$ be a Klein surface with boundary of genus $g$ with a group of automorphisms $G$. If $p$ divides $o(G)$, then $o(G) \leqslant 4(g-1)$.

Proof. Let $o(G)=p k$. Since $3 \nmid(p-1), p k \leqslant 8(g-1)=8 p$ by Lemma 
4 , and $k \leqslant 8$. Let $S$ be a $p$-Sylow subgroup of $G$. Since $k \leqslant 8<p, S$ is normal in $G$. By Lemma $5, X / S$ is of genus 2 and the quotient map $X \rightarrow X / S$ is unramified. Moreover by Lemma $7, G / S$ acts as a group of automorphisms of $X / S$. Since $o(G / S)=k$, Lemma 6 shows that the only possibilities for $k$ are $1,2,3,4,6,8$.

Let $C$ be the centralizer of $S$ in $G$. By a theorem of Burnside [11, p. 137], $S$ has a normal complement $V$ in $C$, so that $C=S \times V$. Let $w=o(V)$ and $q=[G: C]$, so that $o(G)=p q w$. Since $S$ is normal in $G, G / C$ is isomorphic to a subgroup of the automorphism group of $S[11, \mathrm{p}$. 50]. The automorphism group of $S$ is cyclic of order $p-1$. Thus $q \mid(p-1)$. But $q w=k$. From the hypothesis $(p-1) / 2$ and 6 are relatively prime. Then from the possibilities for $k$ we see that either $q=1$ or $q=2$.

Now consider the action of $C=S \times V$ on $X$. We have the following commutative diagram.

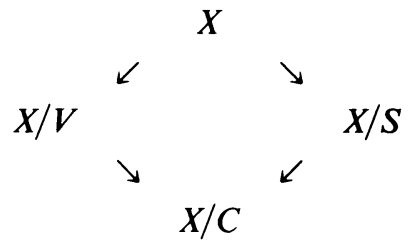

The quotient map $X \rightarrow X / S$ is an unramified $p$-sheeted covering. The quotient map $X / V \rightarrow X / C$ arises from the action of a cyclic group of order $p$. If this map were ramified, then an element of the cyclic group would fix some point of $X / V$. Then, since $o(V)$ and $p$ are relatively prime, we could find an element of order $p$ in $C$ that fixes a point of $X$. But there is no such element. Therefore the map $X / V \rightarrow X / C$ is an unramified $p$-sheeted covering. Now the Hurwitz ramification formula shows that the genus of $X / C$ is not zero.

If $q=1$, then $G=C$ and $o(G) \leqslant 4(g-1)$ by Lemma 2 . If, on the other hand, $q=2$, then $w=\frac{1}{2} k \leqslant 4$ and $V$ is abelian. Then $C$ is also abelian, and $o(G)=2 \cdot o(C) \leqslant 2 \cdot 2(g-1)=4(g-1)$ by Lemma 3. This completes the proof.

Recall that the closed disc $D$ is the Klein surface of genus 0 with nonempty boundary; $D$ has (up to isomorphism) a unique dianalytic structure $[2$, p. 60].

Now suppose that $p$ is a prime such that $p \equiv 11(\bmod 12)$. Let $g-1=p$, and let $X$ be a Klein surface with boundary of genus $g$ with a group of automorphisms $G$. Suppose $o(G) \geqslant 4 g$. Then $p \nmid o(G)$ by Theorem 1. Also, by Lemma 2 , the quotient space $X / G=D$. The quotient map $\pi: X \rightarrow D$ is ramified above, say, $\omega$ points, $a_{1}, \ldots, a_{\omega}$, with ramification indices $k_{1}, \ldots$, $k_{\omega}$. We refer to [7, pp. 205-207] for the possibilities for $\omega$ with $o(G) \geqslant 4 g$. These are
A. $\omega=2$
$a_{1}, a_{2} \in D^{\circ}$
$k_{1}=2, k_{2}=3$
B. $\omega=3$
$k_{1}=k_{2}=2, k_{3}=3$
C. $\omega=4$
$a_{1}, a_{2} \in \partial D, a_{3} \in D^{\circ}$
$k_{1}=k_{2}=2, k_{3}=3,3 \leqslant k_{4} \leqslant 5$
D. $\omega=4$
$a_{1}, a_{2}, a_{3}, a_{4} \in \partial D$
$k_{1}=k_{2}=k_{3}=2,3 \leqslant k_{4}$. 
Both cases A and B give $o(G)=6(g-1)$; these then are ruled out by Theorem 1. Thus the quotient map $\pi$ is ramified above 4 boundary points of $D$, and $k_{1}=k_{2}=2$. The Hurwitz ramification formula is

$$
\frac{2 g-2}{o(G)}=1-\frac{1}{k_{3}}-\frac{1}{k_{4}}
$$

Suppose $k_{3}=3,3 \leqslant k_{4} \leqslant 5$. If $k_{4}=3$, then $o(G)=6(g-1)$ which again is ruled out by Theorem 1 . If $k_{4}=4$, then $(2 g-2) / o(G)=5 / 12$ or $24(g-1)=24 p=5 \cdot o(G)$. But $p \nmid o(G)$ and $p>5$, so this case cannot occur. Similarly $k_{4}=5$ is not a possibility. Thus none of the cases with $k_{3}=3$ occur.

Therefore $k_{3}=2$ and $k_{4} \geqslant 3$. Now write $k=k_{4}$. We have

$$
\frac{2 p}{o(G)}=\frac{2 g-2}{o(G)}=\frac{1}{2}-\frac{1}{k}=\frac{k-2}{2 k} \text {. }
$$

Thus $4 k p=(k-2) \cdot o(G)$. Since $p$ does not divide $o(G)$, we have $p \mid(k-2)$. In particular, $k-2 \geqslant p=g-1$, and $k \geqslant g+1$. Then

$$
\frac{2 g-2}{o(G)} \geqslant \frac{1}{2}-\frac{1}{g+1}=\frac{g-1}{2(g+1)}
$$

Therefore $4(g+1) \geqslant o(G)$.

Combining these calculations with the example of $\$ 2$, we have the following theorem.

TheORem 2. Let $p$ be a prime such that $p \equiv 11(\bmod 12)$. If $g=p+1$, then $\nu(g)=4(g+1)$.

COROLlaRY. For an infinite number of values of the genus $g, \nu(g)=4(g+1)$.

The corollary follows from Dirichlet's general theorem on primes in an arithmetic series.

The values of $g$ less than 100 to which Theorem 2 applies are 12, 24, 48, 60, 72 , and 84.

4. Nonorientable surfaces. We still assume that $p$ is a prime such that $p \equiv 11(\bmod 12)$, and that $X$ is a Klein surface with boundary of genus $g=p+1$ with a group of automorphisms $G$. As before, let $o(G) \geqslant 4 g$. We continue to use the notation of the discussion preceding Theorem 2 .

First note that the bound $4(g+1)$ is attained with $k=k_{4}=g+1$. Otherwise $k-2 \geqslant 2 p=2 g-2$ and $k \geqslant 2 g$. Then

$$
\frac{2 g-2}{o(G)} \geqslant \frac{1}{2}-\frac{1}{2 g}=\frac{g-1}{2 g}
$$


and $o(G) \leqslant 4 g$. Thus no group with order between $4 g$ and $4(g+1)$ can act on $X$.

Now let us examine more closely the case in which the bound $4(g+1)$ is attained. Suppose $o(G)=4(g+1)$, so that the quotient map $\pi: X \rightarrow D$ is ramified above 4 boundary points $a_{1}, a_{2}, a_{3}, a_{4}$, the ramification indices being $k_{1}=k_{2}=k_{3}=2, k_{4}=g+1$. The fiber above $a_{4}$ consists of two interior points of $X$; call these points $x$ and $y$. $G$ contains a subgroup $H$ of index 2 that fixes $x$, and an element $\varphi$ such that $\varphi(x)=y$. Since $H$ is normal in $G, H=\varphi H \varphi^{-1}$ is also the subgroup of $G$ that fixes $y . H$ contains a cyclic group $K$ of order $g+1$ (find a dianalytic chart $(U, z)$ at $x$ such that $h(U)=U$ for all $h \in H$ and let $K=\left\{h \in H \mid z \circ h \circ z^{-1}\right.$ is analytic $\}$ ). The ramification index $k_{4}=o(K)=g+1$. For more details, see [2, pp. 52-54]. Let $K$ act on $X$, and let $Z=X / K$ be the quotient space, $\mu: X \rightarrow Z$ the quotient map. The covering map $\mu$ is ramified above two interior points of $Z$, the ramification index being $g+1$ in each fiber. There is no other ramification since $o(K)$ $=p+2$ is odd. Let $\delta$ be the genus of $Z$. The Hurwitz ramification formula gives

$$
\frac{2 g-2}{g+1}=2 \delta-2+2\left(1-\frac{1}{g+1}\right)+2\left(1-\frac{1}{g+1}\right)=2 \delta+\frac{2 g-2}{g+1}
$$

Therefore $\delta=0$ and $Z$ is the disc $D$. Now the quotient map $\mu: X \rightarrow D$ is ramified only above interior points of $D$. By a result of [9], $X$ is orientable ( $X$ is not folded along $\partial D$, so it is possible to lift the orientation of $D$ to define an orientation of $X)$. Thus the bound $4(g+1)$ is attained only for orientable surfaces. We have the following result for nonorientable surfaces.

TheORem 3. Let $p$ be a prime such that $p \equiv 11(\bmod 12)$. Suppose $X$ is a nonorientable Klein surface with boundary of genus $g=p+1$. If $G$ is a group of automorphisms of $X$, then $o(G) \leqslant 4 g$.

Finally we construct for each $g$ a nonorientable surface with boundary that has an automorphism group of order $4 g$.

ExAmple. For any integer $g \geqslant 2$, let $Y$ be a sphere with $2 g$ holes, with the holes placed around the equator, centered around the vertices of an inscribed regular polygon with $2 g$ sides. $Y$ has a group of orientation-preserving automorphisms isomorphic to the dihedral group $D_{2 g}$. The antipodal map $\tau: Y \rightarrow Y$ is an orientation-reversing automorphism. The automorphism group of $Y$ is isomorphic to $C_{2} \times D_{2 g}$.

Let $W=Y / \tau$ be the quotient space. $W$ is a real projective plane with $g$ holes, a nonorientable Klein surface of genus $g$. The dihedral group $D_{2 g}$ acts as a group of automorphisms of $W$.

Thanks are due the referee for calling our attention to [5] and [10]. 


\section{REFERENCES}

1. R. D. M. Accola, On the number of automorphisms of a closed Riemann surface, Trans. Amer. Math. Soc. 131 (1968), 398-408. MR 36 \#5333.

2. N. L. Alling and N. Greenleaf, Foundations of the theory of Klein surfaces, Springer-Verlag, Berlin and New York, 1971.

3. M. Heins, On the number of 1-1 directly conformal maps which a multiply-connected plane region of finite connectivity $p(>2)$ admits onto itself, Bull. Amer. Math. Soc. 52 (1946), 454-457. MR 8, 21.

4. A. Hurwitz, Ubber algebraische Gebilde mit eindeutigen Transformationen in sich, Math. Ann. 41 (1893), 403-442.

5. Takao Kato, On the number of automorphisms of a compact bordered Riemann surface, Kōdai Math. Sem. Rep. 24 (1972), 224-233. MR 46 \#5610.

6. C. Maclachlan, A bound for the number of automorphisms of a compact Riemann surface, J. London Math. Soc. 44 (1969), 265-272. MR 38 \# 4674.

7. C. L. May, Automorphisms of compact Klein surfaces with boundary, Pacific J. Math. 59 (1975), 199-210.

8. Large automorphism groups of compact Klein surfaces with boundary. I, Glasgow Math. J. 18 (1977), 1-10.

9. _- Cyclic automorphism groups of compact Klein surfaces with boundary (to appear).

10. K. Oikawa, Notes on conformal mappings of a Riemann surface onto itself, Kōdai Math. Sem. Rep. 8 (1956), 23-30; A supplement to "Notes on conformal mappings of a Riemann surface onto itself”, ibid. 8 (1956), 115-116. MR 18, 290; 797.

11. W. R. Scott, Group theory, Prentice-Hall, Englewood Cliffs, N.J., 1964. MR 29 \#4785.

12. R. Tsuji, On conformal mapping of a hyperelliptic Riemann surface onto itself, Kōdai Sem. Rep. 10 (1958), 127-136. MR 20 \#6521.

Department of Mathematics, University of TeXas, Austin, TeXas 78712

Current address: Department of Mathematics, University of Kentucky, Lexington, Kentucky 40506 